\title{
Ultrasound- and Temperature-Induced Gelation of Gluconosemicarbazide Gelator in DMSO and Water Mixtures
}

\author{
Mothukunta Himabindu and Aruna Palanisamy * \\ Polymers and Functional Materials Division, Indian Institute of Chemical Technology (CSIR-IICT), \\ Hyderabad, Telangana 500007, India; binduhimachemistry@gmail.com \\ * Correspondence: aruna@iict.res.in; Tel.: +91-044-27191447 \\ Academic Editor: Xian Jun Loh \\ Received: 10 February 2017; Accepted: 12 April 2017; Published: 18 April 2017
}

\begin{abstract}
We have developed amphiphilic supramolecular gelators carrying glucose moiety that could gel a mixture of dimethyl sulfoxide (DMSO) and water upon heating as well as ultrasound treatment. When the suspension of gluconosemicarbazide was subjected to ultrasound treatment, gelation took place at much lower concentrations compared to thermal treatment, and the gels transformed into a solution state at higher temperatures compared to temperature-induced gels. The morphology was found to be influenced by the nature of the stimulus and presence of salts such as $\mathrm{KCl}, \mathrm{NaCl}, \mathrm{CaCl}_{2}$ and surfactant (sodium dodecyl sulphate) at a concentration of $0.05 \mathrm{M}$. The gel exhibited impressive tolerance to these additives, revealing the stability and strength of the gels. Fourier transform infrared spectroscopy (FTIR) revealed the presence of the intermolecular hydrogen bonding interactions while differential scanning calorimetry (DSC) and rheological studies supported better mechanical strength of ultrasound-induced (UI) gels over thermally-induced (TI) gels.
\end{abstract}

Keywords: amphiphilic gelators; ultrasound; thermoreversible; hydrogen bonding; gluconohydrazide

\section{Introduction}

The synthesis of low molecular weight building blocks from renewable resources for the development of soft [1,2] materials through supramolecular concept is of great interest. The products of this synthesis include many attractive properties, such as their inherent biodegradability, non-toxicity, and eco-friendliness with structural diversity, all of which lend them wider applications in chemistry, biochemistry and material sciences. To date, several methods have been employed to synthesize effective hydro and organogels by utilizing renewable plant-derived sources through supramolecular concept. Carbohydrate centered low-molecular weight gelators (LMWGs), which can be functionalized explicitly to yield biocompatible gels through a self-assembly process, find many biomedical applications [3,4]. There are also many reports on low molecular weight sugar-based amphiphilic [5,6] and bola amphiphilic gels [7] in which sugar polar head groups are held by linkages such as ester, ether, amine and urea between the hydrophilic head and hydrophobic tail groups in order to facilitate well-defined and stable macroscopic structures by secondary interactions [8-13]. They form a physically crosslinked network by self-aggregation through hydrogen bonding, $\pi-\pi$ stacking, solvophobic interactions and van der Waals interactions [14-20]. The dynamic reversible nature of these interactions depends on the gelator structure, which gives these LMWGs the inherent ability to exhibit phase transition changes triggered by external stimuli, including ultrasound [21], temperature [22], $\mathrm{pH}$ [23], and light [24]. These materials have attracted increasing attention due to their wide applications in drug release [25], sensors [26], and catalysis [27]. 
Among these stimuli, low frequency ultrasound is a powerful therapeutic tool used in molecular imaging and in releasing drug molecules in tumor-targeted regions [28]. To initiate the aggregation of molecules that leads to the formation of highly stable 3D network through ultrasound stimuli, balancing hydrogen bonds with other physical interactions is the prerequisite for the gelling action. This induces changes in the morphology, and the orientation of microscopic surface features such as surface wettability [29-31]. Ultrasound is a novel method that often influences the mode of molecular self-assembled morphologies due to change in hydrogen bonding from intramolecular to intermolecular interactions [32]. Jiaju $\mathrm{Xu}$ described $\mathrm{CuBuPc}$ organogel formation assisted by ultrasound treatment and the fabrication of transistors by the doctor blading technique, which significantly increased the charge carrier mobility due to stronger $\pi-\pi$ interactions providing a way for the development of electronic devices [33]. Dou et al. described a urea-based gelator which dissolved in polar aprotic solvents at elevated temperatures, in which a precipitate was generated upon cooling the hot solution to room temperature. Further gels were obtained by sonicating the hot solution in a water bath for $10 \mathrm{~s}$ [34]. Navneet Goyal et al. have explored a series of sugar-derived pH responsive gelators, and studied the Naproxen release kinetics by entrapping this non-steroidal anti-inflammatory drug in a dimethyl sulfoxide (DMSO)/water mixture gel under neutral and acidic conditions [35].

In this contribution, we report the synthesis and gelation properties of a thermoreversible amphiphilic sugar-based gelator in a mixture of $\mathrm{H}_{2} \mathrm{O} / \mathrm{DMSO}(20 / 80 \mathrm{v} / \mathrm{v})$. DMSO is a polar organic solvent miscible with water and other aqueous solvents. DMSO is used as a drug vehicle both in vivo and in vitro experiments. It is also used as an effective alternative cancer cure. Hence, the gelation of a mixture of DMSO and water finds many applications in the area of drug release. The amphiphilic gelator, with a hydrophilic glucose moiety as the head group and a hydrophobic tail connected by polar semi carbazide linkages, was able to immobilize a mixture of polar aprotic solvent and water. More interestingly, different morphologies were observed for the gels obtained by heat-cooling method and ultrasound treatment-resting method. The head group with multiple hydroxyl groups and a tail end comprising of alkyl chains tagged through urea-type linkages self-aggregates into continuous folded sheets. A combination of spectroscopic methods, X-ray diffraction technique and microscopic methods were used to arrive at a hypothesis for the molecular arrangement of the gels induced by heat and ultrasound.

\section{Results and Discussion}

The gelator was found to gel a mixture of polar aprotic solvents DMSO, NMP ( $N$-methyl 2-pyrrolidone), DMF ( $N, N$-dimethyl formamide), DMAc ( $N, N$-dimethyl acetamide) and water at a concentration of $20-25 \mathrm{mg} / \mathrm{mL}$ at $(80 / 20 v / v)$ (Table S1). However, we have specifically chosen the DMSO/water gels for further studies since DMSO has already been reported to be used as a drug vehicle. The gel obtained by the ultrasound-induced (UI-gels) was stable at room temperature and upon heating it transformed to a solution state. This hot solution converted to a gel upon cooling to ambient temperature. The ultrasound-induced gelation occurred at relatively less concentrations and gelation time than the thermally-induced gelation (TI-gels), facilitating the formation of continuous folded sheets as shown in Figure 1. Gelation occurred in the presence of salts such as $\mathrm{NaCl}, \mathrm{KCl}, \mathrm{CaCl}_{2}$ and SDS (sodium dodecyl sulphate) at $0.05 \mathrm{M}$ concentration. The presence of different ions in the solvent led to an increase in the gelation time [36]. 


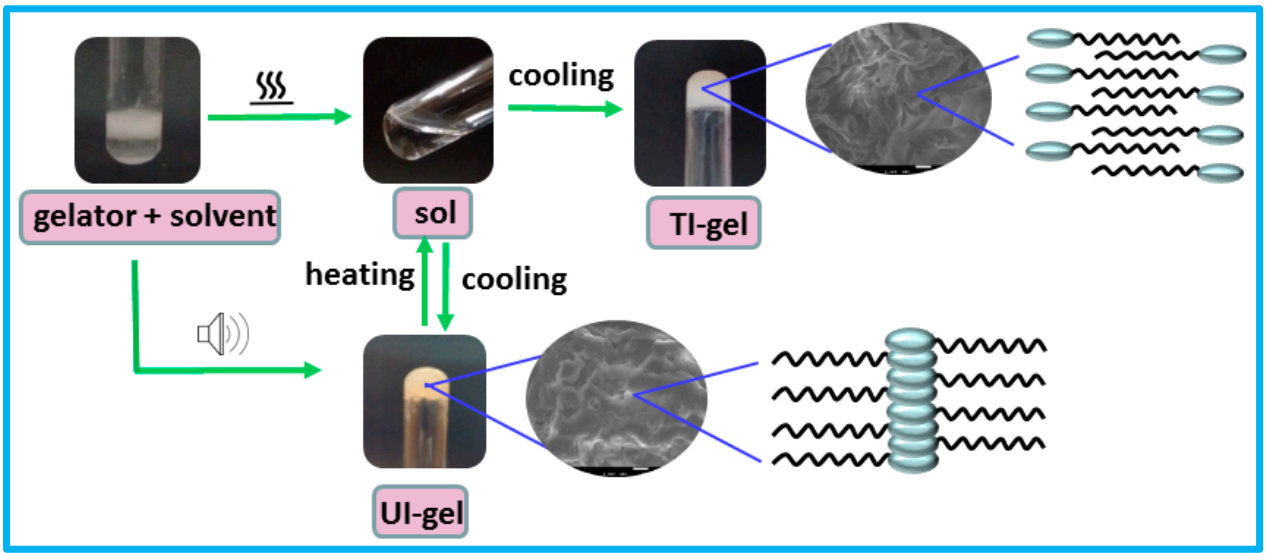

Figure 1. Digital photographs of sol gel transition of ultrasound- and thermally-induced gels.

\subsection{Fourier transform infrared spectroscopy (FT-IR)}

Low molecular weight gelators self-assemble into a three-dimensional network and restrict the flow of bulk fluid. The driving forces responsible for the three-dimensional network is built by intermolecular interactions like hydrogen bonding in most of the urea-type molecules. To probe the change in hydrogen bonding interactions with respect to the nature of the stimuli, IR spectra were recorded for the TI-gels and UI-gels of the gelator. The spectrum of the gelator (Figure 2) in the solution phase exhibits peaks at $3432 \mathrm{~cm}^{-1}$ (NH stretching frequency) and $1654 \mathrm{~cm}^{-1}$ ( $>\mathrm{C}=\mathrm{O}$ stretching frequency) ascribed to non-hydrogen bonded $\mathrm{NH}$ and carbonyl stretching frequencies. In the gel state, the NH band shifted to $3423 \mathrm{~cm}^{-1}$ (TI-gels) and $3417 \mathrm{~cm}^{-1}$ (UI-gels) and the carbonyl band shifted to $1647 \mathrm{~cm}^{-1}$ (TI-gels) and $1640 \mathrm{~cm}^{-1}$ (UI-gels), confirming the involvement of hydrogen bonding between $>\mathrm{C}=\mathrm{O}$ and amide $\mathrm{N}-\mathrm{H}$ groups in the gel state. In the case of the UI-gels, the extent of shifting of peaks was more compared to the TI-gels, indicating a greater extent of $\mathrm{H}$ bonding in the UI-gels.

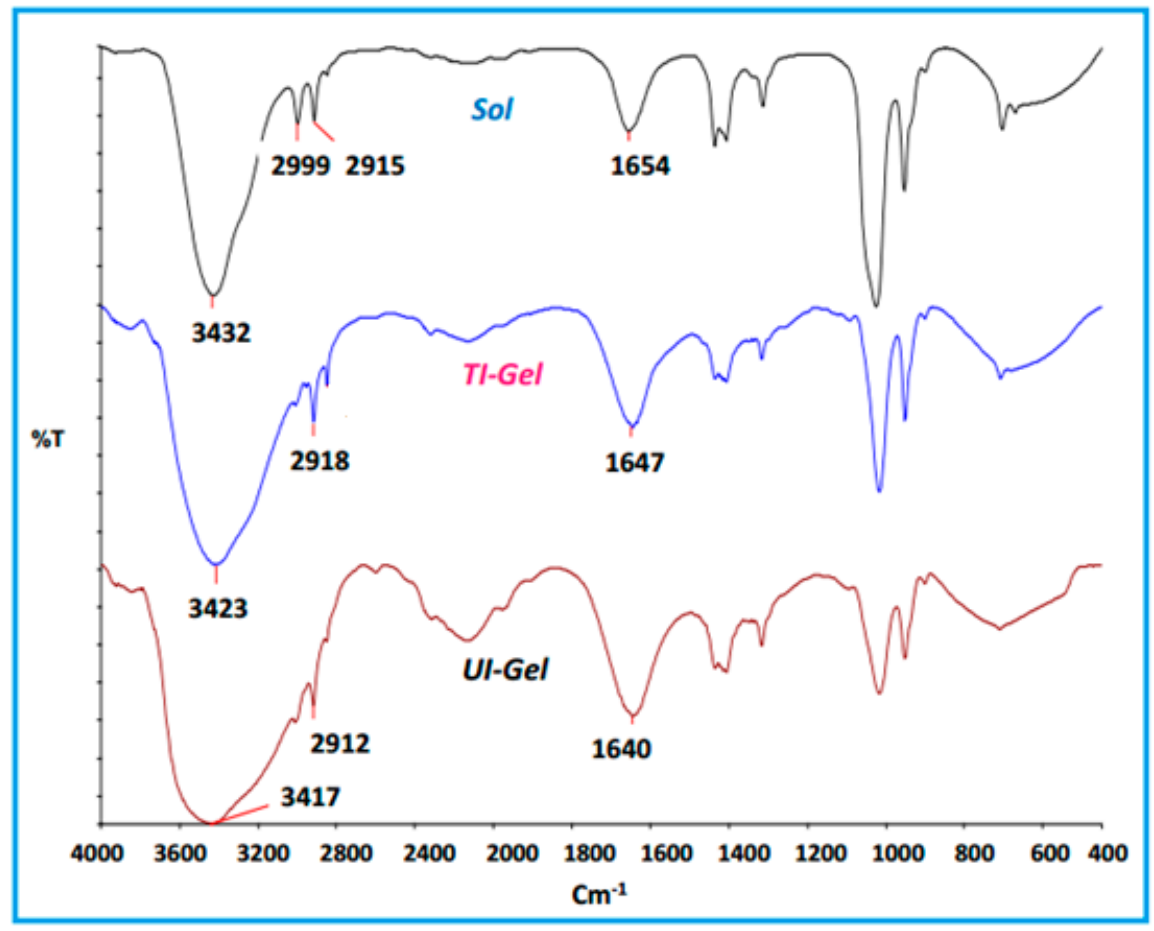

Figure 2. FTIR spectrum of sol, thermally-induced (TI) gel and ultrasound-induced (UI) gel of the gelator in dimethyl sulfoxide (DMSO)/water mixtures $(80 / 20 \mathrm{v} / \mathrm{v}$ ) at a concentration of $30 \mathrm{mg} / \mathrm{mL}$. 


\subsection{Thermal Properties}

To understand the thermal stability of the amphiphile in the self-assembled phase, differential scanning calorimetry (DSC) was employed to study the phase transition of the gels. The transitions are dependent on the concentration of the gelator, as well as the nature of the external stimuli. The DSC curves of TI-gel and UI-gel are presented in Figure 3, which shows a marked difference in the melting temperature of the both the gels. Thermal-induced gel exhibits a gel melting temperature at $101{ }^{\circ} \mathrm{C}$, whereas UI gel shows transition at $111{ }^{\circ} \mathrm{C}$. The higher melting temperature in case of UI-gel may be because of the mode of aggregation of the molecules through intermolecular hydrogen bonding interactions between hydrophilic head groups. These gels require more heat to disrupt the physical crosslinks in the gel phase, which are strengthened by secondary interactions. In case of thermally-induced gels, it is assumed that the alkyl chains are oriented and stacked one above the other and the energy required for disrupting the interactions is less. Thus, the nature of the stimuli has a dramatic influence on the aggregation behaviour and melting of the gels.

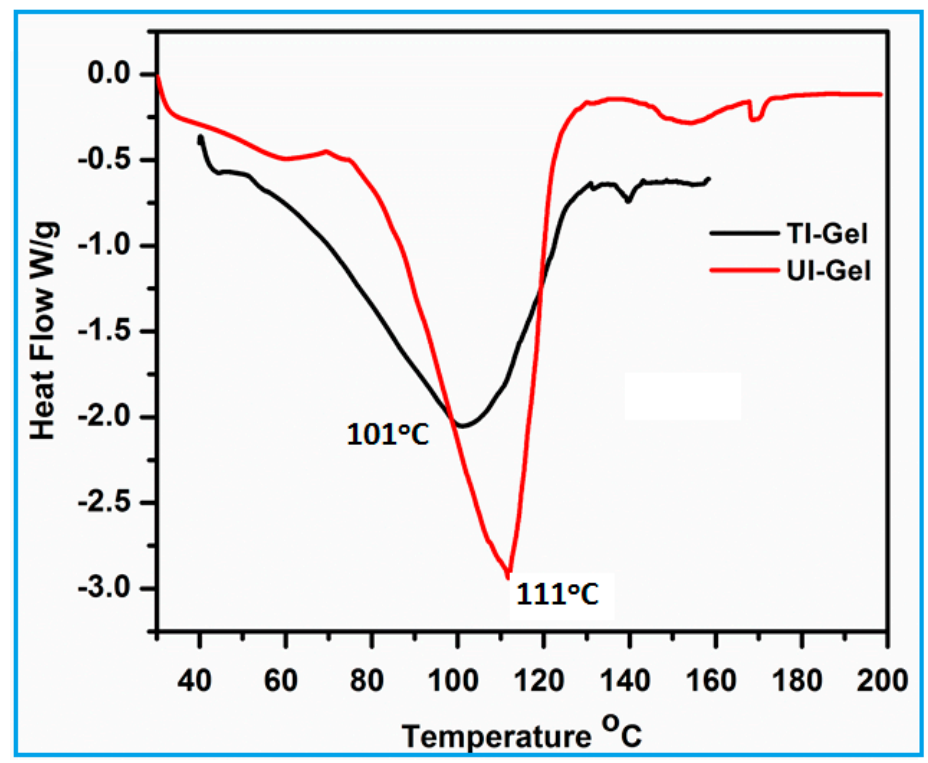

Figure 3. Thermogram of UI-gel and TI-gel in DMSO/water $(80 / 20 v / v)$ at $30 \mathrm{mg} / \mathrm{mL}$ concentration.

\subsection{Morphology}

To estimate the microstructural changes of molecular aggregation upon sonication, heating, the influence of salts like $\mathrm{KCl}, \mathrm{NaCl}$, and $\mathrm{CaCl}_{2}$ at a concentration of $0.05 \mathrm{M}$, and surfactant viz. sodium dodecyl sulphate (SDS), morphological analysis was carried out by Scanning Electron Microscopy (SEM). SEM images (Figure 4) of the the xerogels of DMSO/water mixtures obtained from thermal induction showed continuous folded sheets of aggregates in a 3D network, and some of them are comprised of entangled and fused networks of sheets. Micrographs of ultrasound-induced xerogels shows a flake-like morphology. During the sonication process, the amphiphilic molecules arrange in a perfect order to enhance intermolecular hydrogen bonding interactions. This distinct microlevel morphology suggests that sonication plays a key role in directing the aggregates. It is known that presence of salts in the gelator favours the formation of charges on the head group of amphiphiles by reducing the electrostatic repulsions between the head groups, rendering the solubility in aqueous solvents [37]. However, the presence of small concentrations of salts and surfactants did not bring any considerable morphological changes in which the polar head groups favoured tight packing of the molecules through different modes of aggregations, leading to such distinct microscopic features [38,39]. Instead, the gels were found to be stable and their morphology intact with the 
addition of salts, an attractive property of the gels since stability of the gels in biological fluids is essential for biomedical applications.

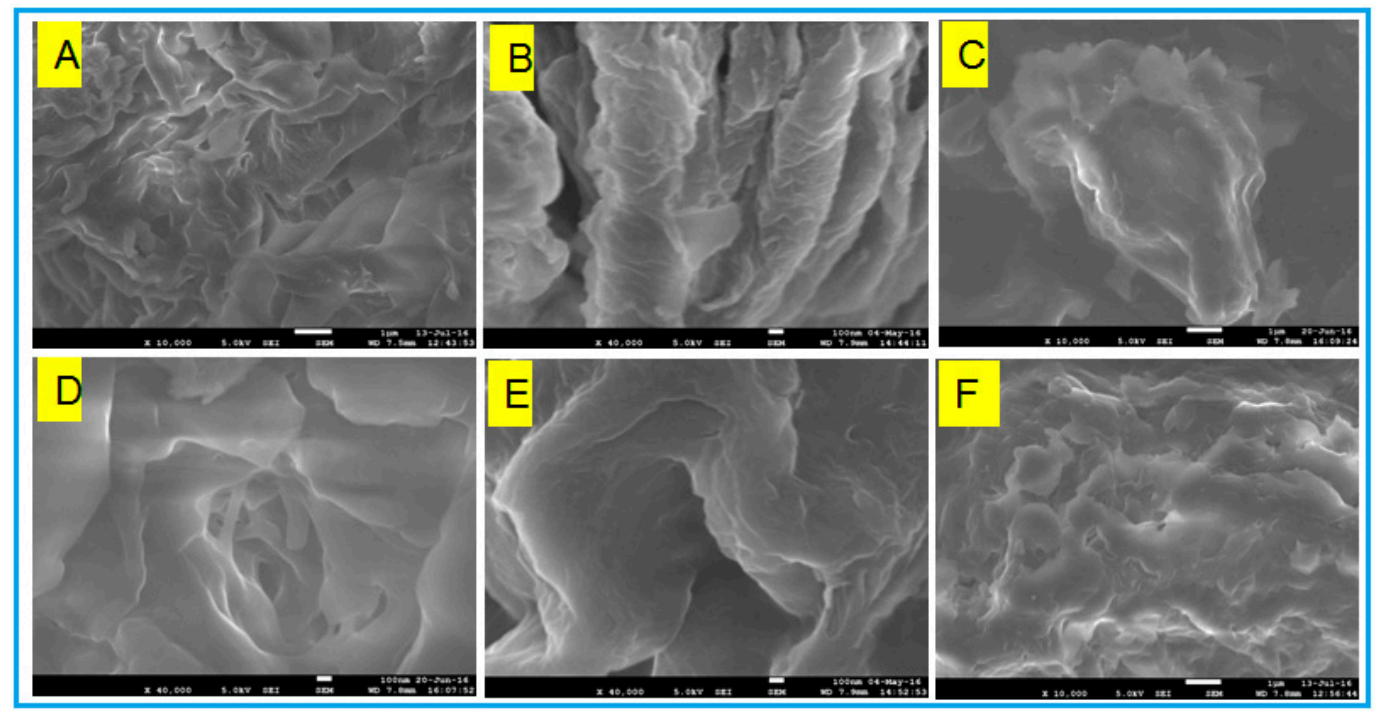

Figure 4. SEM images of xerogels of Gluconosemicarbazide gelator in DMSO/water mixture at a concentration of $30 \mathrm{mg} / \mathrm{mL}$ under different stimuli: (A) thermally-induced (1 $\mu \mathrm{m})$; (B) SDS (100 nm); (C) $\mathrm{KCl}(1 \mu \mathrm{m}) ;(\mathbf{D}) \mathrm{NaCl}(100 \mathrm{~nm}) ;(\mathbf{E}) \mathrm{CaCl}_{2}(100 \mathrm{~nm}) ;$ (F) ultrasound-induced $(1 \mu \mathrm{m})$.

\subsection{Rheological Properties}

To gain further insight into the dynamic mechanical properties of the gelators, at a constant temperature $\left(25^{\circ} \mathrm{C}\right)$ TI-gels and UI-gels were subjected to continuous frequency sweep and amplitude sweep to confirm their viscoelastic properties.

In the frequency sweep measurements at a fixed amplitude of stress, both storage modulus $\left(G^{\prime}\right)$ and loss modulus $\left(G^{\prime \prime}\right)$ of the UI-gels were found to be greater than those of the TI-gels, with linear response over the entire plateau region. It is evident from Figure 5a that storage modulus and loss modulus is invariant for all of the systems in entire frequency range with $G^{\prime}>G^{\prime \prime}$, suggesting the gel behavior and the stability of the system. Furthermore, to analyze the strength and stability of the ultrasound-induced and thermal-induced gels, $G^{\prime}$ of the UI-gel is found to be greater than that of $G^{\prime}$ of the TI-gel. This is due to the reinforcing action of intermolecular hydrogen bonding interactions induced by sonication [40].

In the amplitude sweep experiments, shear stress is applied to the sample sinusoidally. The response of the stress is shown in Figure 5b, where storage modulus $G^{\prime}>G^{\prime \prime}$ (loss modulus) during the initial application of shear stress. Both the gels were found to withstand lower stresses at which storage modulus exceeds loss modulus, and upon increasing the shear stress the magnitude of $G^{\prime}$ starts decreasing with the reduction in the stored energy and begins to flow. At this stage the networks in the gel phase suffer a collapse by which gel disruption arises, and $G^{\prime}$ subsequently crosses over $G^{\prime \prime}$ with $G^{\prime \prime}>G^{\prime}$ referred to as yield stress. The behavior of the gels in the vicinity of yield stress indicates the transition from gel state to sol state. Ultrasound-treated gel has a higher yield stress $(\sigma y=108 \mathrm{~Pa})$ than the thermally-induced gel $(\sigma \mathrm{y}=23 \mathrm{~Pa})$. This implies that sonicated gel has higher mechanical strength than thermally-treated gel [41]. These results are consistent with DSC and SEM data, as discussed earlier. 


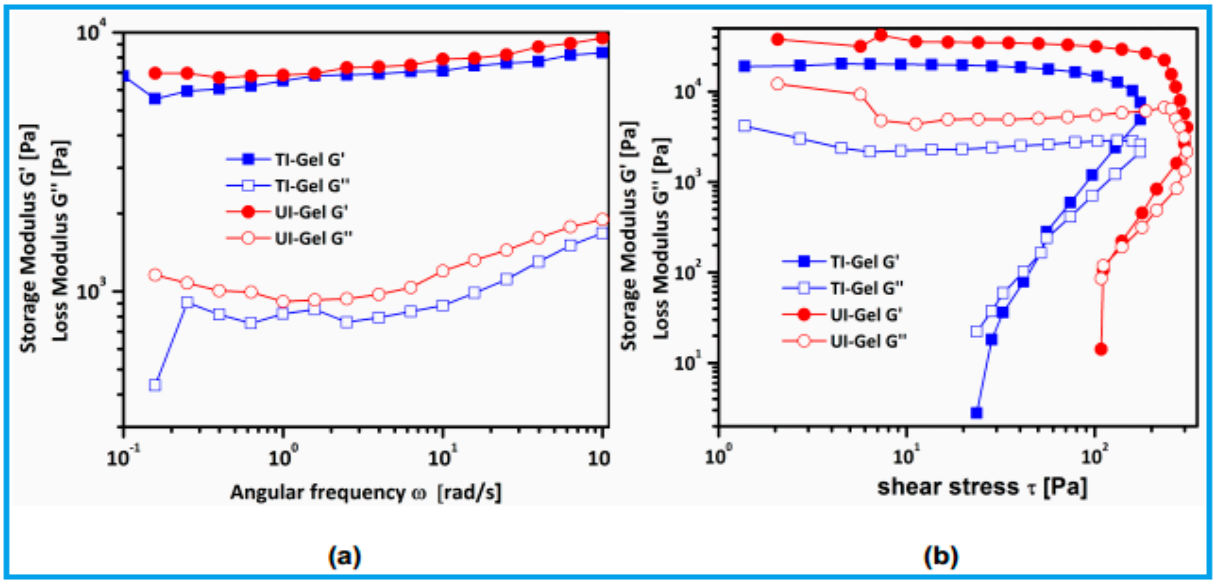

Figure 5. Storage modulus $G^{\prime}$ and loss modulus $G^{\prime \prime}$ as a function of (a) angular frequency; (b) shear stress for gels at a concentration of $30 \mathrm{mg} / \mathrm{mL}$.

\subsection{Mode of Packing in the Xerogels}

To obtain deep insight into the aggregation mode arising from both sugar moiety and alkyl chains, wide angle XRD was employed. Figure $6 \mathrm{a}, \mathrm{b}$ show the X-ray pattern of the gelator in the xerogel state, prepared from the DMSO/water mixtures under different conditions i.e., ultrasound and thermal treatments (Figure 6a), from freeze-thawing method at a concentration of $30 \mathrm{mg} / \mathrm{mL}$. A very intense signal at $4.1 \AA$ in both of the gels corresponds to the plane of the hydrogen bonding [42]. The diffractogram (Figure $6 \mathrm{~b}$ ) of the gelator obtained after ultrasound irradiation shows the series of peaks at $13.4,6.5$, and $4.6 \AA$, following a ratio of $1: 1 / 2: 1 / 3$, which suggests that molecules are arranged in a layered structure in the gelator. The Braggs distance in the low angle region at the $\mathrm{d}$ spacing 13.49 and $14.17 \AA$ (UI- and TI-gels) corresponds to extended molecular length of the gelators in which the molecules are arranged in bilayer organization by intra and interlayer hydrogen bonding and electrostatic interactions. Based on the type of aggregation, the molecular length of the self-assembled gelators would vary as reflected in the different $d$ spacing values. A lower value is expected for UI-gels, due to the association of molecules through head group stacking. In TI-gels, interdigitated arrangement is expected in addition to the aggregation of head groups as proposed by John et al. [2,43]. In Figure $6 \mathrm{a}$, peaks at 3.5 and $3.3 \AA$ are assigned to the distance between the alkyl chains within the molecules and distance between the molecules along the hydrogen bonding plane, which is not found in case of the ultrasound-induced gelator suggesting that alkyl chains are loosely packed in the aggregates with head-to-head arrangement, as shown in Figure 1.

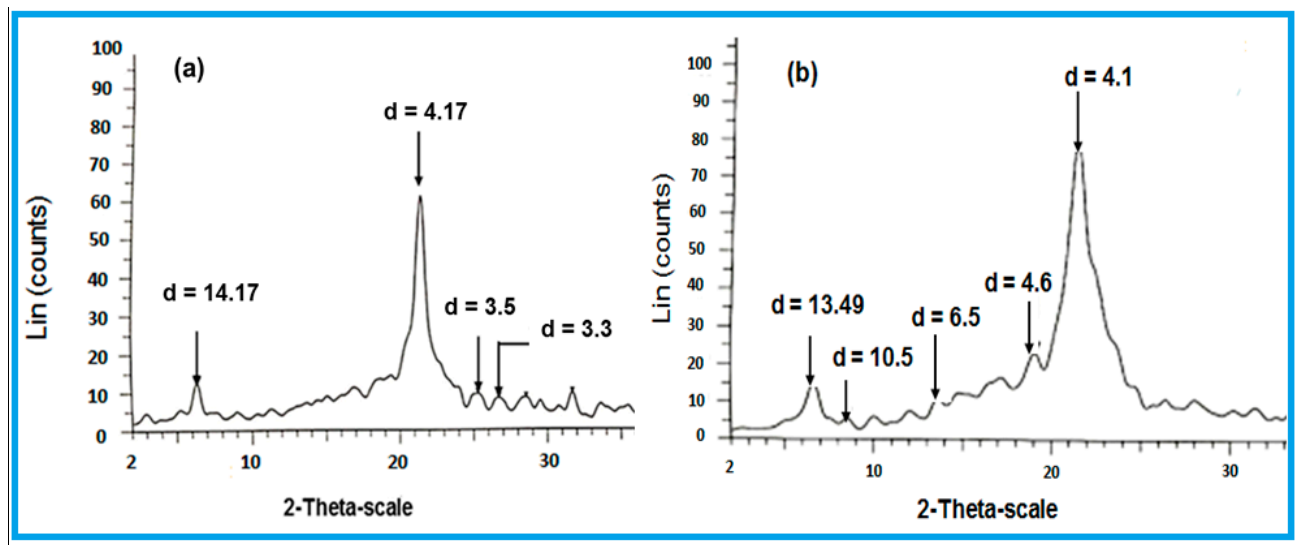

Figure 6. X-ray diffraction spectrum of xerogels of (a) thermally-induced gel and (b) ultrasoundinduced gel. 


\section{Conclusions}

In this study, we report gluconosemicarbazide gelator with a hydrophilic glucose head and a hydrophobic fatty acid tail which could gelate a mixture of DMSO and water $(80 / 20 v / v)$ both upon heating and ultrasonication. The thermally-reversible gels thus obtained were found to be stable in the presence of $0.05 \mathrm{M} \mathrm{KCl}, \mathrm{NaCl}, \mathrm{CaCl}_{2}$ and surfactant (sodium dodecyl sulphate). The different modes of aggregation arising due to the stimuli-induced gelation gives rise to different morphologies and behavior. The extent of intermolecular hydrogen bonding in ultrasound-treated gels is more compared to thermally-induced gels as inferred from FTIR, differential scanning calorimetry and rheological studies. The rheological studies also confirmed that the UI-gels are stronger than the TI-gels due to higher gelator-gelator intermolecular hydrogen bonding interactions which impart high strength that facilitates less dissipation of energy during the shear process.

\section{Experimental}

\subsection{Materials}

Glucono $\delta$-lactone was purchased from Sigma Aldrich (Milwaukee, WI, USA). Hydrazine hydrate, octanoyl chloride, decanoyl chloride, lauryl chloride, myristoyl chloride, palmitoyl chloride, methanol, sodium azide and benzene were procured from SDFCL, Mumbai, India.

\subsection{Synthesis of Gluconohydrazide}

Delta gluconolactone ( $5 \mathrm{~g}$, 1 equiv. is dissolved in $80 \mathrm{~mL}$ of methanol, and hydrazine hydrate $\left(1.3 \mathrm{~mL} 1.5\right.$ equiv.) is added. This mixture is stirred for $5 \mathrm{~h}$ at $70^{\circ} \mathrm{C}$. This reaction mixture is left standing at $0{ }^{\circ} \mathrm{C}$ for some time and the precipitate is subsequently filtered off, washed with cold methanol, then washed with ether and dried in vacuum at $45{ }^{\circ} \mathrm{C}$. The structure of the gluconohydrazide was confirmed by ${ }^{1} \mathrm{H}$ NMR (Figure S1).

${ }^{1} \mathrm{H}$ NMR, DMSO, $500 \mathrm{MHz}(\delta$ in ppm): $8.7(\mathrm{~S}, 1 \mathrm{H}), 5.21-5.25(\mathrm{~d}, J=5.4 \mathrm{~Hz}, 1 \mathrm{H}), 4.45-4.55$ $(\mathrm{dd}, J=5.4 \mathrm{~Hz}, 1 \mathrm{H}), 4.30-4.40(\mathrm{dd}, 2 \mathrm{H}), 4.21(\mathrm{~S}, 2 \mathrm{H}) 3.48-3.91(\mathrm{~m}, 1 \mathrm{H}) 3.99-4.05(\mathrm{t}, 1 \mathrm{H})$.

\subsection{Preparation of Alkyl Isocyanate from Alkyl Acyl Azide}

Palmitoyl chloride ( $2 \mathrm{~g}, 1$ equiv.) was added to $50 \mathrm{~mL}$ of acetone in a double neck RB and sodium azide ( $0.68 \mathrm{~g}, 1.45$ equiv.), dissolved in $20 \mathrm{~mL}$ of water, was added dropwise to the acyl chloride mixture while maintaining a temperature of $10-15^{\circ} \mathrm{C}$. The reaction was continued up to $2 \mathrm{~h}$ at room temperature, and an organic layer containing acyl azide was separated. To this azide, benzene was added and refluxed at $80^{\circ} \mathrm{C}$ for $1 \mathrm{~h}$. The course of the reaction was followed by infrared analysis. The appearance of the isocyanate peak at $2264 \mathrm{~cm}^{-1}$ and the disappearance of the azide peak around $2142 \mathrm{~cm}^{-1}$ confirmed the formation of the corresponding isocyanate.

\subsection{Synthesis of Amphiphilic Glucono Semicarbazide Gelator}

Alkyl isocyanate ( $1 \mathrm{~g}, 1$ equiv.) dissolved in $20 \mathrm{~mL}$ of anhydrous DMF was added to a double neck RB. Gluconohydrazide (1.4 g, 1.2 equiv.) dissolved in $10 \mathrm{~mL}$ of anhydrous DMF was added to the reaction mixture and stirred at $60{ }^{\circ} \mathrm{C}$ for $2 \mathrm{~h}$. The reaction was monitored by IR for the disappearance of the isocyanate peak. The reaction mixture was then poured into water and the precipitate was filtered and vacuum-dried at $50{ }^{\circ} \mathrm{C}$ overnight. Schematic representation for the synthesis of gluconosemicarbazide gelator is shown in Figure 7. The structure of the compound was confirmed by ${ }^{1} \mathrm{H}$ NMR and ${ }^{13} \mathrm{C}$ NMR (Figures S2 and S3).

${ }^{1} \mathrm{H}$ NMR, DMSO, $500 \mathrm{MHz}(\delta$ in ppm): $9.23(\mathrm{~S}, 1 \mathrm{H}), 7.66(\mathrm{~S}, 1 \mathrm{H}), 5.35-5.39(\mathrm{~d}, J=5.3 \mathrm{~Hz}, 1 \mathrm{H}), 4.66-4.73$ $(\mathrm{dd}, J=5.3 \mathrm{~Hz}, 1 \mathrm{H}), 4.44-4.56(\mathrm{dd}, J=5.4 \mathrm{~Hz}, 2 \mathrm{H}), 4.24-4.29(\mathrm{t}, 1 \mathrm{H}), 4.08-4.12(\mathrm{t}, 1 \mathrm{H}), 3.94-4.03(\mathrm{~m}, 1 \mathrm{H})$ 2.92-3.02 (m, 2H), 1.33-1.43 (m, 2H), 1.19-1.32 (m, 24H), 0.82-0.88 (t, 3H). 
${ }^{13} \mathrm{C}$ NMR, DMSO, $300 \mathrm{MHz}$ ( $\delta$ in ppm): 171 (C6), 157 (C7), 72.3 (C2), 71.2 (C5), 71.1 (C4), 70.4 (C3), 63 (C1), 30.8 (C8), 29.2 (C12), 28.6 (C9), 28.55 (C11), 28.2, 25.9 (C10), 21.6 (C13), 13.4 (C14).

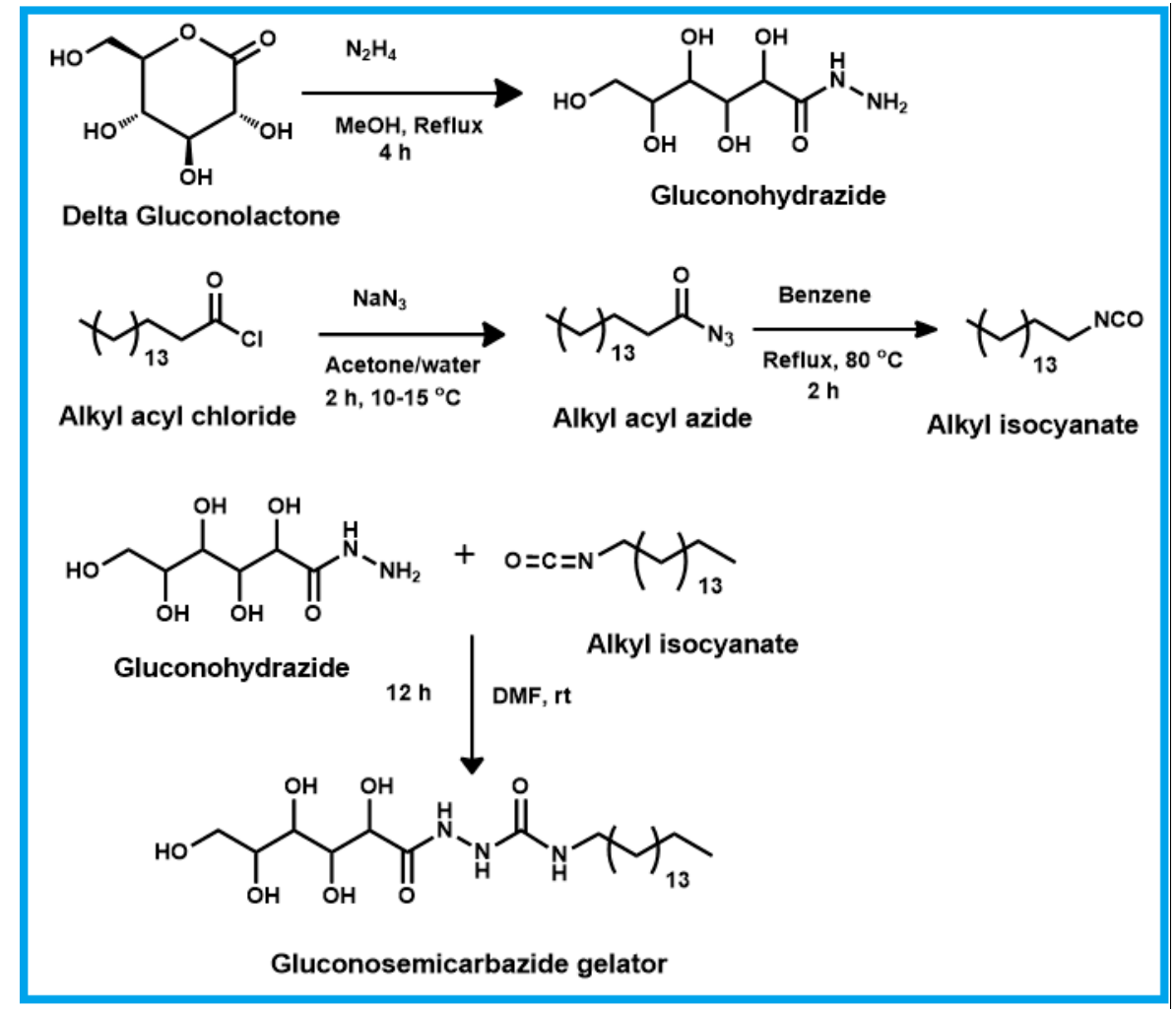

Figure 7. Schematic route to synthesize gluconosemicarbazide gelator.

\subsection{Gelation Tests}

To confirm, gelation test tube inversion method was employed. A weighed amount of the gelator was added to a mixture of organic solvents (polar aprotic) such as DMSO, NMP, DMF and water in a test tube vial and subjected to heat at an elevated temperature to obtain a homogenous solution. This hot solution, upon cooling, turned to a solid aggregate mass and was found to be stable upon inverting the test tube. Further, the melting temperature of the gels $\left(T_{\text {gel }}\right)$ was determined by the dropping ball method, wherein steel balls were placed on the surface of the gels held in a test tube and heated at $1{ }^{\circ} \mathrm{C} / \mathrm{min}$ using a heating block mantle. The temperature at which the ball reached the bottom of test tube was considered as the gel melting temperature.

In addition, the compound showed gelation when the mixture of organic solvents and water was subjected to sonication at $30^{\circ} \mathrm{C}$ for a period of $4-5 \mathrm{~min}$.

\subsection{Instrumentation}

\subsubsection{Proton NMR}

${ }^{1} \mathrm{H}$ NMR spectra was recorded in DMSO-d6 on a BRUKER $500 \mathrm{MHz}$ NMR spectrometer (Bremen, Germany) and tetramethylsilane (TMS) was used as an internal reference.

\subsubsection{FT-IR Spectroscopy}

FT-IR measurements on gels prepared from a DMSO $/ \mathrm{H}_{2} \mathrm{O}$ mixture and the nature of the gelator-solvent interaction was determined by IR spectroscopy using a Perkin Elmer, spectrum 100 FT-IR spectrometer (PerkinElmer, Waltham, MA, USA). The gel and the solution of the gelator in $\mathrm{DMSO} / \mathrm{H}_{2} \mathrm{O}$ solvent were taken on $\mathrm{KBr}$ discs and scanned in the $4000-400 \mathrm{~cm}^{-1}$ range. 


\subsubsection{Differential Scanning Calorimetry}

The gel melting temperatures were determined by DSC Q 100 series (TA instruments, New Castle, DE, USA) from room temperature to $100^{\circ} \mathrm{C}$ at a heating rate of $5{ }^{\circ} \mathrm{C} / \mathrm{min}$ under a nitrogen atmosphere (flow rate $50 \mathrm{~mL} / \mathrm{min}$ ). Weighed quantity of the gel $(12 \mathrm{mg}$ ) was taken in a hermitic pan and sealed.

\subsubsection{X-ray Diffraction}

The xerogels for XRD were obtained by freeze-drying the gels at $-100^{\circ} \mathrm{C}$. X-ray diffraction spectra for the xerogels were obtained by using a Siemens/D-5000 X-ray diffractometer (Munich, Germany) using $\mathrm{Cu}$ Ka radiation of wavelength $1.54 \AA$ and a continuous scan speed of $0.045 / \mathrm{min}$. Diffraction data were recorded at room temperature, in the range of $2^{\circ} \leq 2 \theta \leq 65^{\circ}$.

\subsubsection{Scanning Electron Microscopy (SEM)}

SEM images of the xerogels were taken on a Scanning Electron Microscope-Energy Dispersive Spectrometer (SEM-EDS), HITACHI S-3400N (Tokyo, Japan). The accelerating voltage was $15 \mathrm{kV}$, and the emission was $10 \mathrm{Ma}$.

\subsubsection{Oscillatory Rheological Measurements}

Rheological measurements were performed with a strain-controlled rheometer (series Modular Compact Rheometer 102, Anton Paar's, Graz, Austria-Europe) equipped with a parallel plate. The gap distance was fixed at $1 \mathrm{~mm}$. To determine the storage moduli, $G^{\prime}$, and loss moduli, $G^{\prime \prime}$, the measured angular frequency $\omega$, ranged from 0.1 to $100 \mathrm{~s}^{-1}$ and the applied shear strain amplitude was $0.5 \%$ in the linear viscoelastic regime measured at room temperature $\left(25^{\circ} \mathrm{C}\right)$, at the same time the strain amplitude sweep was performed at a constant frequency $(f) 1.0 \mathrm{~Hz}$ with strain ranging from 0.01 to 100 , at $25{ }^{\circ} \mathrm{C}$.

Supplementary Materials: The following are available online at www.mdpi.com/2310-2861/3/2/12/s1. Figure S1. ${ }^{1} \mathrm{H}$ NMR spectrum of gluconohydrazide gelator. Figure S2. ${ }^{1} \mathrm{H}$ NMR spectrum of gluconosemicarbazide gelator. Figure S3. ${ }^{13} \mathrm{C}$ NMR spectrum of gluconosemicarbazide gelator in DMSO- $\mathrm{d}_{6}$. Table S1. Gelation properties of $\mathrm{C}_{16}$ gelator [solvent:water $=80: 20(v / v)$ ].

Acknowledgments: Mothukunta Himabindu acknowledges University Grants Commission for the senior research fellowship.

Author Contributions: Aruna Palanisamy conceived and designed the experiments; Mothukunta Himabindu performed the experiments and analyzed the samples. Both the authors, Aruna Palanisamy and Mothukunta Himabindu interpreted the data and drafted the manuscript.

Conflicts of Interest: The authors declare no conflicts of interest.

\section{References}

1. Vemula, P.K.; John, G. Crops: A green approach toward self-assembled soft materials. Acc. Chem. Res. 2008, 41, 769-782. [CrossRef] [PubMed]

2. John, G.; Vemula, P.K. Design and development of soft nanomaterials from biobased amphiphiles. Soft Matter. 2006, 2, 909-914. [CrossRef]

3. Wang, W.; Wang, H.; Ren, C.; Wang, J.; Tan, M.; Shen, J.; Yang, Z.; Wang, P.G.; Wang, L. A saccharide-based supramolecular hydrogel for cell culture. Carbohydr. Res. 2011, 346, 1013-1017. [CrossRef] [PubMed]

4. Vemula, P.K.; Wiradharma, N.; Ankrum, J.A.; Miranda, O.R.; John, G.; Karp, J.M. Prodrugs as self-assembled hydrogels: A new paradigm for biomaterials. Curr. Opin. Biotechnol. 2013, 24, 1174-1182. [CrossRef] [PubMed]

5. Bühler, G.; Feiters, M.C.; Nolte, R.J.; Dötz, K.H. A metal-carbene carbohydrate amphiphile as a lowmolecular-mass organometallic gelator. Angew. Chem. Int. Ed. 2003, 42, 2494-2497. [CrossRef] [PubMed]

6. Nandi, S.; Altenbach, H.J.; Jakob, B.; Lange, K.; Ihizane, R.; Schneider, M.P.; Gün, U.; Mayer, A. Amphiphiles based on D-glucose: Efficient low molecular weight gelators. Org. Lett. 2012, 14, 3826-3829. [CrossRef] [PubMed] 
7. Latxague, L.; Gaubert, A.; Maleville, D.; Baillet, J.; Ramin, M.A.; Barthélémy, P. Carbamate-based bolaamphiphile as low-molecular-weight hydrogelators. Gels 2016, 2, 25. [CrossRef]

8. Fuhrhop, J.H.; Helfrich, W. Fluid and solid fibers made of lipid molecular bilayers. Chem. Rev. 1993, 93, 1565-1582. [CrossRef]

9. Jung, J.H.; Rim, J.A.; Han, W.S.; Lee, S.J.; Lee, Y.J.; Cho, E.J.; Kim, J.S.; Ji, Q.; Shimizu, T. Hydrogel behavior of a sugar-based gelator by introduction of an unsaturated moiety as a hydrophobic group. Org. Biomol. Chem. 2006, 4, 2033-2038. [CrossRef] [PubMed]

10. Mathiselvam, M.; Loganathan, D.; Varghese, B. Synthesis and characterization of thiourea-and urea-linked glycolipids as low-molecular-weight hydrogelators. RSC Adv. 2013, 3, 14528-14542. [CrossRef]

11. Goyal, N.; Cheuk, S.; Wang, G. Synthesis and characterization of D-glucosamine-derived low molecular weight gelators. Tetrahedron 2010, 66, 5962-5971. [CrossRef]

12. Sankarprasad, B.; Hyean Kim, B. An insulin-sensing sugar-based fluorescent hydrogel. Chem. Commun. 2006, 17, 1842-1844.

13. Wang, G.; Cheuk, S.; Yang, H.; Goyal, N.; Reddy, P.N.; Hopkinson, B. Synthesis and characterization of monosaccharide-derived carbamates as low-molecular-weight gelators. Langmuir 2009, 25, 8696-8705. [CrossRef] [PubMed]

14. Hafkamp, R.J.; Feiters, M.C.; Nolte, R.J. Organogels from carbohydrate amphiphiles. J. Org. Chem. 1999, 64, 412-426. [CrossRef]

15. Jung, J.H.; John, G.; Masuda, M.; Yoshida, K.; Shinkai, S.; Shimizu, T. Self-assembly of a sugar-based gelator in water: Its remarkable diversity in gelation ability and aggregate structure. Langmuir 2001, 17, 7229-7232. [CrossRef]

16. Ohsedo, Y.; Oono, M.; Saruhashi, K.; Watanabe, H. N-Alkylamido-D-glucamine-based gelators for the generation of thixotropic gels. RSC Adv. 2014, 4, 48554-48558. [CrossRef]

17. Jung, J.H.; Shinkai, S.; Shimizu, T. Spectral Characterization of self-assemblies of aldopyranoside amphiphilic gelators: What is the essential structural difference between simple amphiphiles and bolaamphiphiles? Chem. Eur. J. 2002, 8, 2684-2690. [CrossRef]

18. Yan, N.; He, G.; Zhang, H.; Ding, L.; Fang, Y. Glucose-based fluorescent low-molecular mass compounds: Creation of simple and versatile supramolecular gelators. Langmuir 2009, 26, 5909-5917. [CrossRef] [PubMed]

19. Clemente, M.J.; Romero, P.; Serrano, J.L.; Fitremann, J.; Oriol, L. Supramolecular hydrogels based on glycoamphiphiles: Effect of the disaccharide polar head. Chem. Mater. 2012, 24, 3847-3858. [CrossRef]

20. Minakuchi, N.; Hoe, K.; Yamaki, D.; Ten-no, S.; Nakashima, K.; Goto, M.; Mizuhata, M.; Maruyama, T. Versatile supramolecular gelators that can harden water, organic solvents and ionic liquids. Langmuir 2012, 28, 9259-9266. [CrossRef] [PubMed]

21. Avalos, M.; Babiano, R.; Cintas, P.; Gómez-Carretero, A.; Jimenez, J.L.; Lozano, M.; Ortiz, A.L.; Palacios, J.C.; Pinazo, A. A family of hydrogels based on ureido-linked aminopolyol-derived amphiphiles and bolaamphiphiles: Synthesis, gelation under thermal and sonochemical stimuli, and mesomorphic characterization. Chem. Eur. J. 2008, 14, 5656-5669. [CrossRef] [PubMed]

22. Cui, J.; Liu, A.; Guan, Y.; Zheng, J.; Shen, Z.; Wan, X. Tuning the helicity of self-assembled structure of a sugar-based organogelator by the proper choice of cooling rate. Langmuir 2009, 26, 3615-3622. [CrossRef] [PubMed]

23. Zhou, S.L.; Matsumoto, S.; Tian, H.D.; Yamane, H.; Ojida, A.; Kiyonaka, S.; Hamachi, I. pH-responsive shrinkage/swelling of a supramolecular hydrogel composed of two small amphiphilic molecules. Chem. Eur. J. 2005, 11, 1130-1136. [CrossRef] [PubMed]

24. Clemente, M.J.; Tejedor, R.M.; Romero, P.; Fitremann, J.; Oriol, L. Maltose-based gelators having azobenzene as light-sensitive unit. RSC Adv. 2012, 2, 11419-11431. [CrossRef]

25. Giammanco, G.E.; Sosnofsky, C.T.; Ostrowski, A.D. Light-Responsive Iron (III)-Polysaccharide Coordination Hydrogels for Controlled Delivery. ACS Appl. Mater. Interfaces 2015, 7, 3068-3076. [CrossRef] [PubMed]

26. Zhang, C.; Losego, M.D.; Braun, P.V. Hydrogel-based glucose sensors: Effects of phenylboronic acid chemical structure on response. Chem. Mater. 2013, 25, 3239-3250. [CrossRef]

27. Zheng, Y.; Wang, A. Ag nanoparticle-entrapped hydrogel as promising material for catalytic reduction of organic dyes. J. Mater. Chem. 2012, 22, 16552-16559. [CrossRef] 
28. Rapoport, N.Y.; Kennedy, A.M.; Shea, J.E.; Scaife, C.L.; Nam, K.H. Controlled and targeted tumor chemotherapy by ultrasound-activated nano emulsions/microbubbles. J. Control. Release. 2009, 138, 268-276. [CrossRef] [PubMed]

29. Wu, J.; Yi, T.; Shu, T.; Yu, M.; Zhou, Z.; Xu, M.; Zhou, Y.; Zhang, H.; Han, J.; Li, F.; et al. Ultrasound switch and thermal self-repair of morphology and surface wettability in a cholesterol-based self-assembly system. Angew. Chem. 2008, 120, 1079-1083. [CrossRef]

30. Zhang, Y.; Ding, H.; Wu, Y.; Zhang, C.; Bai, B.; Wang, H.; Li, M. Ultrasound-induced controllable morphology and growth dimension in a dihydrazide-based self-assembly system. Soft Matter. 2014, 10, 8838-8845. [CrossRef] [PubMed]

31. Park, S.M.; Kim, B.H. Ultrasound-triggered water gelation with a modified nucleoside. Soft Matter. 2008, 4, 1995-1997. [CrossRef]

32. Wang, Y.; Zhan, C.; Fu, H.; Li, X.; Sheng, X.; Zhao, Y.; Xiao, D.; Ma, Y.; Ma, J.S.; Yao, J. Switch from intra-to intermolecular $\mathrm{H}$-bonds by ultrasound: Induced gelation and distinct nanoscale morphologies. Langmuir 2008, 24, 7635-7638. [CrossRef] [PubMed]

33. Xu, J.; Wang, Y.; Shan, H.; Lin, Y.; Chen, Q.; Roy, V.A.; Xu, Z. Ultrasound-induced organogel formation followed by thin film fabrication via simple doctor blading technique for field-effect transistor applications. ACS Appl. Mater. Interfaces 2016, 8, 18991-18997. [CrossRef] [PubMed]

34. Dou, C.; Wang, C.; Zhang, H.; Gao, H.; Wang, Y. Novel urea-functionalized quinacridone derivatives: Ultrasound and thermo effects on supramolecular organogels. Chem. Eur. J. 2010, 16, 10744-10751. [CrossRef] [PubMed]

35. Goyal, N.; Mangunuru, H.P.; Parikh, B.; Shrestha, S.; Wang, G. Synthesis and characterization of pH responsive D-glucosamine based molecular gelators. Beilstein J. Org. Chem. 2014, 10, 3111-3121. [CrossRef] [PubMed]

36. Srivastava, A.; Ghorai, S.; Bhattacharjya, A.; Bhattacharya, S. A tetrameric sugar-based azobenzene that gels water at various $\mathrm{pH}$ values and in the presence of salts. J. Org. Chem. 2005, 70, 6574-6582. [CrossRef] [PubMed]

37. Pal, A.; Shrivastava, S.; Dey, J. Salt, $\mathrm{pH}$ and thermoresponsive supramolecular hydrogel of $\mathrm{N}$-(4-N-tetradecyloxybenzoyl)-L-carnosine. Chem. Commun. 2009, 45, 6997-6999. [CrossRef] [PubMed]

38. Grillo, I.; Kats, E.I.; Muratov, A.R. Formation and growth of anionic vesicles followed by small-angle neutron scattering. Langmuir 2003, 19, 4573-4581. [CrossRef]

39. Zhai, L.; Zhao, M.; Sun, D.; Hao, J.; Zhan g, L. Salt-induced vesicle formation from single anionic surfactant SDBS and its mixture with LSB in aqueous solution. J. Phys. Chem. B 2005, 109, 5627-5630. [CrossRef] [PubMed]

40. Baddi, S.; Madugula, S.S.; Sarma, D.S.; Soujanya, Y.; Palanisamy, A. Combined Experimental and computational study of the gelation of cyclohexane-based bis(acyl-semicarbazides) and the multi-stimuliresponsive properties of their gels. Langmuir 2016, 32, 889-899. [CrossRef] [PubMed]

41. Das Mahapatra, R.; Dey, J. Ultrasound-induced gelation of organic liquids by L-cysteine-derived amphiphile containing poly(ethylene glycol) tail. Langmuir 2015, 31, 8703-8709. [CrossRef] [PubMed]

42. Moniruzzaman, M.; Sundararajan, P.R. Low molecular weight organogels based on long-chain carbamates. Langmuir 2005, 21, 3802-3807. [CrossRef] [PubMed]

43. Vemula, P.K.; Aslam, U.; Mallia, V.A.; John, G. In situ synthesis of gold nanoparticles using molecular gels and liquid crystals from vitamin-C amphiphiles. Chem. Mater. 2007, 19, 138-140. [CrossRef]

(C) 2017 by the authors. Licensee MDPI, Basel, Switzerland. This article is an open access article distributed under the terms and conditions of the Creative Commons Attribution (CC BY) license (http://creativecommons.org/licenses/by/4.0/). 